\title{
El Programa de Investigación Movimiento de Escuelas EFicaces: Hacia una Perspectiva Basada eN LOS Actores en el ConteXto de AmÉrica Latina*
}

Andrés Sandoval-Hernández Juan Carlos Barrón Pastor

\section{INTRODUCCIÓN}

El Programa de Investigación Movimiento de Escuelas Eficaces (MEE) ha estado trabajando por más de tres décadas en el mejoramiento de la calidad de la educación. Sin embargo, el MEE ha recibido varias críticas relacionadas con aspectos teóricos y metodológicos que han formado parte de un prolífico debate sobre sus límites y potencialidades. El origen y los principales estudios teóricos y metodológicos acerca del MEE coinciden con aquellos realizados en el Reino Unido (RU) y los Estados Unidos de América (EE.UU.) (Mortimore et al., 1988; Rutter et al., 1979) y en otros países desarrollados, de modo que corresponden a los contextos socio-políticos y económicos de países con intereses educacionales similares. Pero en América Latina los contextos sociopolíticos y económicos son muy diferentes de aquellos donde el programa está siendo diseñado y estudiado. Muchas de las críticas que se le hacen al MEE se relacionan con la controvertida conceptualización del contexto social y dichas limitaciones se asocian a enfoques positivistas y a las dificultades para asumir una neutralidad cuando la reproducción de las desigualdades podría verse reforzada debido a esa postura supuestamente imparcial (cf. Slee et al., 1998; Thrupp, 1999; Willmott, 1999). Particularmente, un desafío clave para los programas en América Latina es el que se refiere a la reproducción de las desigualdades. En Latinoamérica la educación es considerada un factor crucial tanto para reproducir como para evitar la reproducción de dichas desigualdades. Más aún, la medición de la eficacia de las escuelas podnía ser especialmente sensible si se consideran los contextos socio-económicos y políticos tal como se ha hecho hasta ahora. Por otra parte, se supone que los investigadores latinoamericanos serían muy receptivos

\footnotetext{
* Este artículo fue traducido del inglés al español por María Eleonora Ramos Vera.
} 
si el mejoramiento de la educación incluyera variables relacionadas con el evitar las desigualdades.

El MEE es una "línea [programa] de investigación que examina las diferencias en el desempeño entre y dentro de las escuelas, como también los factores modificables que mejoran el desempeño escolar, generalmente utilizando las notas de los alumnos para medir este último" (Luyten et al., 2005: 252). Con el fin de lograr una mayor comprensión de dichas diferencias en el desempeño y sus relaciones con factores evaluables que logran mayor eficacia en diferentes contextos, como el de América Latina, es necesario desarrollar contribuciones teóricas y metodológicas que aumentarían sus aplicaciones y ayudarían en el análisis de aquellos conceptos controversiales para eventualmente producir las bases que permitan establecer un nuevo programa de investigación que se adecuaría mejor a la realidad social de América Latina.

Este artículo pretende participar en el debate teórico y metodológico en torno al MEE desde la perspectiva de América Latina utilizando el modelo de análisis de los programas de investigación científica propuesto por Lakatos (1970). Este modelo plantea que este tipo de programas posee un núcleo teórico defendido por un cinturón de argumentos protector, y está sometido a procesos heurísticos donde el debate fortalece o debilita los fundamentos de toda la línea de investigación. Se analizan algunas de las críticas y sus respuestas relacionadas con las concepciones epistemológicas y ontológicas del contexto utilizadas en las investigaciones del MEE, y se proponen algunos aspectos teóricos y metodológicos a considerar en futuras investigaciones cuando se estudian factores del contexto. Se pone énfasis en aquellas críticas que consideran al MEE como esencialmente positivista con el fin de plantear un enfoque alternativo que responda de manera más adecuada a las preocupaciones de América Latina, tales como la necesidad de adaptar las escuelas para reducir en forma efectiva las desigualdades. La idea es mantener algunos aspectos medulares del núcleo del MEE y al mismo tiempo transformar algunos elementos de su tradición positivista en una comprensión más crítica del contexto social.

El planteamiento general que se propone aquí para orientar nuevas investigaciones del MEE en América Latina, y que eventualmente sugiere una nueva base para enfocar los factores contextuales de los programas de investigación, estará basado en la postura original que el MEE gatilló: la escuela sí importa. Es necesario recalcar que no se puede separar a la escuela de su contexto. Por lo tanto, el contexto no es una realidad externa a la escuela como se ha considerado en debates teóricos anteriores. 
Parece obvio que uno de los prerrequisitos de la utilidad de la investigación aplicada es que sus resultados se obtengan del análisis de la realidad donde se implementarán las mejoras. Es decir, si queremos que el MEE sea útil para los países latinoamericanos, es necesario que la investigación de este programa sea adaptada a las escuelas de América Latina y sus características (Murillo, 2003).

En todo el mundo, y particularmente en América Latina, existe la necesidad común de promover contextos de mayor igualdad y destacar el importante papel que pueden jugar las escuelas en impedir o reforzar las desigualdades, así como son clave en términos del acceso de la gente al manejo de la información, de recursos financieros y de remesas. Ignorar las necesidades educacionales debido a las desigualdades contextuales no es una opción (Brunner, 2000), pero si lo es buscar una significativa democratización de las políticas educacionales (Gundara, 2000) y "desenmascarar y deconstruir la aparente neutralidad del civismo -es decir, el conjunto, supuestamente universal y neutral, de valores y prácticas culturales que sostienen la esfera del estado-nación" (May, 1999: 30).

La transferencia de los hallazgos a los sistemas educacionales en América Latina es muy cuestionable si se toma en cuenta la complejidad de las dificultades que experimentan muchas de estas sociedades. La falta general de adecuadas estructuras educacionales y sociales; el hecho de que muchas personas todavía viven en un nivel de subsistencia donde las familias se ven obligadas a recurrir al trabajo infantil como un medio necesario para satisfacer sus necesidades básicas; las formas históricas de desigualdad y la manera en que se entrecruzan con el subdesarrollo post-colonial, son algunos ejemplos de las características actuales en muchos países de la región (Rassool y Morley, 2000). Sin desdeñar lo que se puede aprender de la investigación realizada en Europa y Norteamérica, es necesario evaluar qué aspectos de ella pueden contribuir a la solución de los asuntos latinoamericanos y qué debe desarrollarse a partir del análisis de nuestra propia realidad.

Basándose en lo recién mencionado y con el fin de ayudar a que el MEE logre sus objetivos en el contexto latinoamericano, se debe tener especial interés en determinar, explicar y comparar los patrones de desigualdad en los sistemas educacionales.

La estructura de este documento sigue el modelo de Lakatos: comienza con una breve explicación; luego se presenta el núcleo del MEE mediante la reconstrucción racional ${ }^{2}$ de la tradición central del MEE propuesta con este 
fin por Lauder et al. (1998); después, el artículo sigue la pista de algunas críticas al MEE relacionadas con su enfoque positivista y la falta de una incorporación plausible de factores contextuales; la cuarta sección introduce brevemente algunos argumentos presentados por Teddlie y Reynolds (2001) para defender y mejorar los campos teóricos y metodológicos del MEE. La heurística del artículo propone algunas reflexiones sobre posturas tanto teóricas como metodológicas acerca de la consideración de factores contextuales desde una perspectiva crítica y, al mismo tiempo, mantener algunos de los elementos del núcleo del MEE relacionados con el papel que juegan las escuelas. En las conclusiones realizamos un análisis final del debate del MEE y presentamos nuestra postura.

\section{EL MODELO DE LAKATOS PARA EL ANÁLISIS DE PROGRAMAS DE INVESTIGACIÓN}

De acuerdo con Lakatos (1970), un programa de investigación consta de tres partes principales: un núcleo, un cinturón protector, y una heurística positiva y negativa. El núcleo consiste en hipótesis muy generales que dan al programa de investigación sus características esenciales y supuestos básicos. El cinturón protector es un conjunto de teorías auxiliares que subyacen y protegen el núcleo evitando su falsificación, aunque parte del programa de investigación puede modificarse 0 ajustarse como resultado de anomalías 0 desafíos empíricos sin por ello abandonar el programa mismo. La heurística (positiva y negativa) es un conjunto de normas o indicaciones acerca de cómo manejar el núcleo y el cinturón protector para ayudar al descubrimiento 0 invención. La heurística negativa establece las recomendaciones de lo que no deben hacer los científicos, por ejemplo, que el núcleo del programa no debe abandonarse ni modificarse. Por otra parte, la heurística positiva especifica lo que deberían hacer los científicos dentro del programa -qué asuntos deberían abordar, en qué orden- para cambiar 0 ajustar el cinturón protector.

La principal indicación del mérito de un programa de investigación, según Lakatos, es la medida en que conduce a predicciones confirmadas 0 explicaciones. De esta manera, un programa de investigación progresivo es aquel que mantiene la coherencia entre el núcleo y su cinturón protector y que eventualmente conduce a nuevas predicciones 0 explicaciones, mientras que un programa de investigación degen erativo es el que pierde su coherencia y/o no es capaz de conducir a predicciones o explicaciones innovadoras. 


\section{EL NÚCLEO DEL MEE}

De acuerdo con Lauder et al. (1998: 52-53), una reconstrucción racional de la línea central del MEE sería como sigue:

1. La escuela, en cuanto organización, tiene un efecto sobre los estudiantes, influye sobre el desempeño de los alumnos en los exámenes, en contraposición con la visión de que es el profesor más que la escuela quien produce un efecto sobre el desempeño de los alumnos, o con la perspectiva de que cuando se toman en cuenta los factores contextuales en su totalidad, no hay diferencia significativa entre las escuelas.

2. Estos efectos de la escuela no se producen al azar, y los efectos que mejoran el desempeño escolar en relación al éxito en los exámenes pueden someterse a un proceso de ingeniería sobre la base del MEE.

3. Las escuelas son como cualquiera otra organización donde el personal y los niños responderán a los sistemas, las sanciones y las recompensas de la escuela de modo que es posible lograr una reingeniería exitosa.

4. Las escuelas en tanto organizaciones están estructuradas como organizaciones anidadas. Por ejemplo, su gobierno central/local sirve como la estructura organizacional más amplia dentro de la cual hay diferentes escuelas, departamentos, salas de clase, etc. Además se asume que la eficacia de las escuelas se puede identificar mediante el análisis del desempeño de cada nivel y por la inferencia de la eficacia de la relación entre todos los niveles.

5. Existe un grado de relativa autonomía entre los niveles y entre el sistema educacional y la sociedad. Por lo tanto, las escuelas pueden generar efectos independientes de muchos de los factores externos a la escuela que podrían incidir en los resultados de los exámenes.

\section{ALGUNAS CRÍTICAS EN CONTRA DEL MEE}

Ha habido un largo debate al interior del MEE y un amplio debate entre críticos y defensores de este programa, que merecerían un ensayo por separado. Afortunadamente, existen algunas excelentes compilaciones de críticas y contra críticas. Entre las críticas se destacan los libros de Slee et al. (1998) y Thrupp (1999). En cuanto a las contra críticas, Teddlie y Reynols (2001) escribieron una rigurosa y desapasionada declaración de catorce puntos en defensa del MEE. La obra de Luyten et al. (2005) es otro documento mencionado en este 
artículo ya que es un trabajo reciente que confronta las críticas y recomendaciones relacionadas con este tema. El presente estudio, como se ha dicho anteriormente, se enfoca exclusivamente en aquellos aspectos del debate relacionados con las supuestas posturas positivistas del MEE y con los problemas referidos a conseguir una consideración plausible de factores contextuales en el mismo.

Varios autores critican fuertemente al MEE por su orientación positivista y argumentan en pro de un marco más conceptual y la necesidad de superar lo que llaman la práctica empiricista del MEE (Wyatt, 1996). Estos argumentos generalmente se basan en la dieta reconocida del MEE que se basa en indicadores o factores observables y medibles (Willmott, 1996) y su perspectiva limitada de las complejas relaciones entre las escuelas y sus contextos (Thripp, 1999).

Las criticas señalan que el compromiso del MEE con la metodología positivista necesariamente implica un enfoque simplista de causa-efecto en el estudio de las escuelas eficaces puesto que el positivismo niega que la realidad social sea diferenciada y estructurada, por consiguiente, trata sólo con conjunciones constantes de eventos observables (Willmott, 1999). Como ya se ha mencionado, uno de los objetivos principales del MEE es establecer 0 identificar factores modificables (medibles) que mejoren el desempeño escolar. Estos factores se obtienen al analizar conjuntos de correlaciones entre factores observables directamente y medibles, de modo que se puede decir que aquellos que se correlacionan con un alto desempeño escolar están ciertamente asociados con la eficacia escolar, pero no que la causan, como lo asumen algunas investigaciones del MEE. Se dice que este tipo de argumentación está basado en una literatura 'tecnicista' que carece de una perspectiva crítica sobre la relación entre las escuelas y sus contextos sociales y políticos (Thrupp, 1999).

Otro punto que se ha enfatizado es la ontología atomista esencial que presupone el positivismo. Desde este punto de vista, es casi imposible que el MEE vea a la sociedad como un todo porque tiene muchas limitaciones para reconocer la agencia de la propia escuela y de los actores que la conforman (Willmott, 1996). La escuela no es un sujeto indivisible; es un agente conformado por varios actores que también tienen la facultad de gestión. Las perspectivas basadas en el ser humano que presuponen que las personas son detectores pasivos de determinados hechos involucran explicaciones individualistas de la sociedad (Bhaskar, 1979). En América Latina es mucho más difícil que tales explicaciones resulten convincentes en la medida que esta región del mundo es bastante conocida por ser 'colectivista' (Oxhorn, 
2000). Las perspectivas atomistas tienden a derivarse de dualismos analíticos donde la estructura y la agencia se presentan como dicotomías irreductibles (Archer, 1995, 1996; Layder, 1997), tal como lo es el contexto escolar. Algunos autores han sugerido un enfoque de dualismo cartesiano para explicar cierta interdependencia y al mismo tiempo para reconocer sus propiedades distintivas (Willmott, 1999). Pero estos enfoques fenomenológicos pueden enfrentar problemas al considerar que la sociedad tiene una naturaleza compleja ligada a relaciones de poder (González-Casanova, 2004).

Un buen ejemplo para exponer los problemas de este enfoque se puede encontrar precisamente en la noción de clima escolar. Este es un concepto clave en el cinturón protector del MEE que permite identificar a una gran variedad de factores con la eficacia escolar, organizada alrededor de una noción que supuestamente da cierta cohesión a los ingredientes de la 'receta' para mantenerlos unidos (Lauder et al., 1998). La noción cohesiva de clima admite que éste varía entre las escuelas, y también admite su influencia sobre el papel de los profesores y alumnos; sin embargo, no existe una teoría que explique cómo se crea el clima o cómo interactúa con el desempeño de los actores (Lauder et al., 1998). Tomemos, por ejemplo, el caso de una buena escuela que se esperaría que tuviera altas expectativas de sus alumnos; pero aquellas altas expectativas podrían ser el resultado de haber tenido ingresos de buenos alumnos durante un número de años quienes probablemente obtendrán buenos resultados (Davies, 1997). Otra dificultad que puede observarse en el concepto de clima, y que se advierte comúnmente en otros términos usados por el MEE, es que por lo general ha sido definido por un conjunto de ítems seleccionados arbitrariamente en un cuestionario que varía enormemente entre diferentes estudios (Miller y Fredericks, 1990).

Los autores críticos han demostrado que el hecho de diseñar contextos semejantes hace que su comprensión sea más precisa. Según estas críticas, para atender al problema es necesario cambiar el análisis a un nivel más conceptual que considere mecanismos no observables -como muchos factores contextuales- para explicar fenómenos observables, como las desigualdades (Lauder y Brown, 2007).

\section{EL CINTURÓN PROTECOR DEL MEE}

Esta sección del trabajo se basa principalmente en el artículo escrito por Teddlie y Reynolds (2001) para enfrentar las críticas al MEE contenidas en dos libros: Slee y Weiner con Tomlinson (1998) y Thrupp (1999). El formato del documento corresponde al estilo 'Punto: contrapunto' y presenta una 
serie de 14 críticas al MEE seguidas por las contra críticas, que varían de simples declaraciones de acuerdo a vigorosas defensas.

Teddlie y Reynolds sostienen que las críticas en contra del MEE han sido políticas, metodológicas y teóricas. Tácitamente presentan al MEE como políticamente neutral en la medida que las críticas políticas venían de direcciones muy diferentes. Las críticas metodológicas se presentan principalmente como debates internos del campo y se dice que muchas de ellas se han visto acalladas como resultado de desarrollos metodológicos recientes. Finalmente, concuerdan con las críticas respecto a la falta de bases teóricas; sin embargo, se refieren a los esfuerzos actuales para responder a este problema 'perenne'. En este trabajo, como ya se ha dicho, sólo revisaremos los puntos referentes al discutido positivismo del MEE y su supuesta incapacidad para considerar el contexto de una manera apropiada.

Estos dos autores vindican extensamente que "los investigadores del MEE dan importancia a los factores de contexto" (op. cit.: 58); ellos citan varios estudios donde se han estado considerando explícitamente variables de contexto dentro del MEE desde que se inició este campo, y también mencionan que incluso existen sub-campos de estudio de los factores de contexto en el MEE.

Sin embargo, en vez de reconocer el debate como una lucha recurrente, tal como Thrupp y otros críticos percibinán la discusión sobre el contexto, estos autores prefieren presentar el debate como una prueba de que se ha dado una discusión total en torno al concepto. La evidencia presentada en defensa del MEE deja en claro que sin lugar a dudas el contexto ha sido tomado en cuenta por varios investigadores del MEE. Más aún, ellos también comentan con cierto detalle una cantidad de elementos que se han considerado al realizar 'análisis de contexto' en un trabajo previo dirigido por Teddlie y Stringfiels (1993). La calidad del diseño de investigación utilizado en dicho trabajo es notablemente superior a muchos esfuerzos anteriores y recientes en el campo. Aunque los argumentos presentados por Teddlie y Reynolds respondieron correctamente a las críticas, lo hicieron sólo hasta cierto punto. Está claro que el tipo de trabajo citado por ellos para confrontar la crítica respecto a la falta de modelos sensibles al contexto en el MEE, no es algo común en el programa; y que los investigadores del MEE interesados en diseñar modelos sensibles al contexto, aún tienen mucho que aprender acerca de los mecanismos subyacentes y constitutivos de lo que se conoce como contexto; y acerca de lo que se puede hacer para erradicar el efecto de contextos desfavorables que interfieren sobre el desempeño escolar. 
Es importante mencionar que para autores que asumen perspectivas muy diferentes, incluidos críticos y defensores, la investigación cualitativa es indispensable cuando se piensa seriamente en considerar el contexto para los modelos de eficacia escolar. Es decir, que el MEE se beneficiaría con una mayor cantidad de investigación cualitativa al obtener datos más apropiados para comprender la realidad compleja en la que están sumidas las escuelas y, por consiguiente, útiles para generar teonía en el campo (Lauder et al., 1998; Riddel et al., 1998; Teddlie y Reynolds, 2001; Thrupp, 1999). En todo caso, ambos autores están de acuerdo en que los estudios no podrán aproximarse a la problemática noción de contexto si continúan percibiéndolo como una realidad externa.

Ahora, respecto al supuesto positivismo del MEE, aún cuando Teddlie y Reynolds admiten que algunos investigadores del MEE trabajan con el paradigma positivista, ellos se desligan de las tradiciones (post)positivistas, afirman que han asumido una posición pragmática y sostienen que sus investigaciones tienen las siguientes orientaciones:

- Inclusión de métodos tanto cuantitativos como cualitativos para la recolección y análisis de los datos;

- Uso de una lógica tanto deductiva como inductiva dependiendo de la fase del proyecto de investigación;

- Uso de enfoques epistemológicos tanto objetivos como subjetivos dependiendo de los datos con que se trabaje;

- Una axiología en la cual los valores juegan un gran papel en la interpretación de los resultados;

- Una ontología que acepta una realidad externa, sin embargo niega que la verdad pueda ser determinada en forma absoluta y definitiva.

También argumentan que el Pragmatismo es una orientación filosófica válida para el estudio de las escuelas como lo es la Teoría Crítica (enfoque usado por los críticos). Reiteramos, no se puede discutir que existen algunos trabajos que usan orientaciones filosóficas y una metodología que permiten identificar y comprender mejor aquellos factores que incrementan el desempeño escolar, pero lo que se necesita es una discusión más constructiva y propuestas que conduzcan a la consolidación de toda una metodología que haga avanzar el programa hacia el logro de la meta de mejorar la educación. 


\section{LA HEURÍSTICA}

Hasta aquí se ha presentado un esquema del núcleo del MEE y parte del cinturón protector en relación a algunas de sus limitaciones teóricas y metodológicas referidas a la conceptualización del contexto. En esta sección, el artículo intenta delinear algunas sugerencias acerca de cómo manejar el cinturón protector para fortalecer, cuando sea posible, el núcleo del MEE. La heurística que propone este artículo trata de evitar aquellas contradicciones que adosan limitaciones positivistas al programa, al mismo tiempo que se considera su aplicación en el contexto latinoamericano

En primer lugar, hay que dejar claro que los elementos del núcleo que no deben cambiar son: que las escuelas en tanto organizaciones tienen un efecto sobre los resultados de los alumnos y que los efectos que mejoran el desempeño escolar se pueden obtener sobre la base del MEE en la medida que cuenten con unidades de análisis que permitan a las escuelas reconocer y desarrollar su eficacia interna. Luego, es importante aclarar que nuestros puntos de partida son: i) que la escuela no se encuentra aislada de su contexto; ii) que la educación es fundamental en la perpetuación o restricción de las desigualdades; iii) que los esquemas conductistas de sanciones y premios no pueden ser la base de iniciativas que pretendan mejorar la eficacia de la educación (Lauder et al., 1998); y iv) que aún cuando, por razones de conveniencia, las escuelas se pueden percibir como organizaciones anidadas que pueden ser evaluadas de una manera simplista pero práctica, las escuelas son sistemas históricos complejos y "la configuración [de este tipo de sistemas] está organizada a través de la inter-definición e interacción de relaciones y conjuntos de relaciones" (González-Casanova, 2004: 99) de todos lo actores involucrados.

Los críticos teóricos estarían de acuerdo con Teddlie y Reynolds en cambiar las escuelas, a partir de lo que actualmente son. Pero, ¿en qué sentido deberían cambiar? ¿Quién debería establecer la orientación del cambio? ¿Cómo debería evaluarse la eficacia de aquellas escuelas orientadas hacia mejores sociedades? Estamos de acuerdo en que el cambio escolar es posible dentro de los sistemas socio-políticos existentes en sus condiciones actuales, pero al mismo tiempo aceptamos el planteamiento de los críticos teóricos en cuanto a que la justicia y equidad sociales deben constituir una guía que defina los parámetros del MEE, especialmente en países como los de América Latina, donde la reproducción de las desigualdades no sólo constituye un gran problema de injusticia y violación de los derechos humanos sino también puede constituirse en un problema de paz y seguridad para toda la región. 
Como se ha examinado aquí, el enfoque positivista podría resultar un poco estrecho para el MEE. Sus investigadores están luchando continuamente con el concepto de contexto y varias de sus críticas, como las de Thrupp (1999), han señalado que el problema de la mirada positivista es la explicación teórica de la aparente incapacidad del MEE para entregar conceptos plausibles de factores contextuales. La respuesta a las críticas ha sido que cualquiera orientación filosófica es válida para el estudio de las escuelas, y puede ser cierto, pero el problema radica en que ciertas cosas se pueden explicar mejor y de manera más útil por medio de lentes filosóficos diferentes. Por ejemplo, en la noción de contexto, el problema parece residir en los remanentes de la noción de que la naturaleza de las cosas es una realidad externa. El problema de dar al contexto esta postura ontológica es el argumento principal con que este artículo restringe la línea central del MEE. La escuela y el contexto están intrínsecamente relacionados. Para desarrollar este punto, se seguirán tres líneas: i) el problema de reificar los conceptos; ii) el problema de la agencia de los actores, y iii) el problema de dar a las comunidades escolares el poder de definir y evaluar sus factores contextuales y mantener unidades de análisis generalizables.

El problema de reificar los conceptos. "La idea de 'reificar' se refiere al proceso mental mediante el cual algo se hace fijo, o se transforma en cosa, cuando en realidad es el resultado de un tipo particular de relación social" (How, 2003: 63). Esto es lo que ocurre con la noción de contexto. Una manera muy común de reificar es considerar dos conceptos abstractos y establecer dicotomías entre ellos. En este caso, tanto escuela como contexto son entidades conceptuales y se hace mención a la inter-definición, interacción de relaciones y los conjuntos de relaciones que conforman dichas entidades conceptuales. Si existiera una causa original, ello no sería relevante en los sistemas complejos donde, al verse afectado un aspecto, de alguna manera se verá afectado el sistema completo (Byrne, 1998). Los actores que constituyen la escuela, y la escuela misma, tienen inter-definiciones e interacciones con los actores que dan forma a lo que se denomina contexto. Este es un concepto usado para dar sentido y mantener bajo control algunos aspectos de la realidad.

Tanto las escuelas como los contextos son sistemas complejos en interacción y es posible mejorar el desempeño de uno de los sistemas (la escuela) que afecta y se ve afectado por otro sistema (el contexto). Estos sistemas se diferencian con fines epistemológicos. Realmente no son una dicotomía ya que son entidades conceptuales. Deleuze y Guattari (1972) emplearon la alegoría del rizoma para explicar un modo de organización 'subterránea' que antecede a la entidad conceptual evitando simplificaciones binarias. Un buen ejemplo para tener una imagen de un rizoma es observar las raíces del pasto. 
Es imposible saber qué raíz corresponde a qué pasto, pero cada pasto tiene raíces y todas están interconectadas. Según Deleuze y Guattari, aquellas redes multidimensionales yacen bajo la superficie de todas las entidades conceptuales; así, la escuela y su contexto compartirían de manera rizomática muchos factores, entre ellos la reproducción de las desigualdades. Este punto de vista podría esencialmente estar de acuerdo con diferentes enfoques para explorar la reproducción de las desigualdades en la educación, como los de Bourdieau, Ball, etc.

Lo que parece crucial es la confianza que los autores del MEE puedan tener en la agencia de los actores. ¿Es el contexto algo fijo y fuera del alcance de los actores? ¿ $\mathrm{O}$ es algo que puede ser alcanzado por las personas que constituyen lo que llamamos escuelas? El principal elemento del núcleo del MEE es que las escuelas sí importan. Los investigadores en educación, actores, gestores de políticas y en general cualquiera interesado en la educación, no pueden esperar que se produzca la transformación de todo el mundo social, 0 una discusión de las prioridades de reconocimiento 0 redistribución de otros contextos. Los investigadores y las comunidades escolares tienen agencia, capacidad de gestión. Todos debemos trabajar en ella ahora, con los sistemas socio-políticos existentes. Todos debemos reconocer que nosotros, como parte del contexto, no somos totalmente independientes de las escuelas. Una cosa que podemos hacer es trabajar al interior de las escuelas para proyectar transformaciones contextuales. La escuela es parte del contexto y también lo son los miembros dentro de la escuela. La buena noticia es que esta agencia se puede iniciar en cualquier punto y las comunidades escolares parecen ser un buen agente para hacerlo.

¿Cuán bueno puede llegar a ser? ¿Cuán eficaz? Utilizando este argumento, el trabajo puede realizarse asumiendo que la naturaleza de la escuela está siempre en gestación, y no se relaciona con el contexto como una realidad externa sino como un artefacto humano que potencialmente puede reproducir y transformar la realidad al transformarse a si mismo.

Pero como Deleuze y Guattari lo advirtieron, las determinaciones de las entidades conceptuales -tales como escuela, contexto y eficacia- están codificadas por el poder porque, entre otras explicaciones, aquellos que son poderosos se sienten con la legitimidad para construir identidades e interpretar los deseos de aquellos sobre quienes ejercen hegemonía. Por tanto, si los investigadores usan enfoques interpretativos, podrían estar reproduciendo aquellas dinámicas que supuestamente están tratando de evitar. Esto conduce al segundo problema, el problema de la agencia de los actores. 
El problema de la agencia de los actores (fortaleciendo a los actores). Es fundamental destacar que la construcción de la identidad es un proceso que siempre se produce en relación a otros, y que se pueden vincular estereotipos a los procesos de reproducción de las desigualdades (Barrón-Pastor, 2007). Homi Bhabha puso sobre aviso respecto a que "un rasgo importante del discurso colonial es su dependencia del concepto de 'fijación' en la construcción ideológica de la otredad" (1994: 66). Esta 'fijación' es básicamente un estereotipo, la construcción de identidad no por el otro sino referida a cómo los discursos dominantes construyen aquellas supuestas identidades, donde el 'nosotros' generalmente tiene connotaciones positivas y el 'otros' se asocia frecuentemente con valores negativos.

Es posible caer en estos estereotipos si al definir a los actores en las escuelas no recurrimos a los mismos actores para identificarlos y referimos a ellos. La legitimación de los procesos pasa por la fijación de identidades a través de la construcción ideológica de la otredad (Bhabha, 1994). Las interacciones reforzarían las desigualdades si los 'expertos' construyen el problema, debaten sobre él y determinan un diagnóstico y una solución sin considerar a aquellos que están siendo afectados por aquellas dinámicas. En un trabajo anterior, uno de nosotros concluyó que la 'problematización' de los 'otros' podría ser una 'fase superior de estereotipos' en formas muy complejas (Barrón-Pastor, 2006). Es necesario examinar por medio de trabajos de investigación si el quiebre de los estereotipos se puede lograr profundizando la interacción (Barrón-Pastor, 2007), y si esta interacción puede ser un diálogo 0 no en caso de que la acción comunicativa esté sometida a una racionalidad dominante (Irigaray, 2002).

Así, las culturas dominantes establecen diagnósticos sobre cómo deberían ser las escuelas y cuán importante es el contexto de acuerdo con sus propias referencias culturales. Pero éstas podrían no aplicarse a otras culturas. En esta línea, debido a que el MEE establece los términos de eficacia de las escuelas, este programa de investigación está siendo atacado ya que se sostiene que los problemas y contextos están definidos por un poder dominante que declara neutralidad para poder continuar reproduciendo aquellas dinámicas (Thrupp, 1999). Deleuze y Guattari (1972) explican una dinámica de creación de estereotipos con el argumento Anti-Edipo; en breve, este argumento explica que no es el niño psicótico quien quiere reemplazar al padre sino el padre paranoico quien ve en el niño a un rival. El padre (el dominante) es quien define el contexto social, no el niño (el dominado).

Para América Latina es posible proponer este trastoque teórico. América Latina no pretende sustituir los sistemas educacionales desarrollados 
paranoicamente que reproducen aquellos modelos en que ellos siempre están en la cima; pretendería coexistir de una manera diferente, de acuerdo con su contexto y sus actores. Sería muy probable que la mayoría de los actores latinoamericanos esté de acuerdo en que evitar la reproducción de las desigualdades es una prioridad, y que una necesidad crucial de las escuelas es desarrollar herramientas para hacerlo de la manera más eficaz posible. Parece ser que si enfocamos la noción de contexto como una realidad externa a las escuelas, al mismo tiempo podríamos estar reproduciendo las dinámicas que refuerzan las desigualdades en vez de usar las escuelas como medios de prevención de dichas dinámicas no deseadas.

De manera similar, los actores no les van a quitar el trabajo a los investigadores. Podríamos asegurar que los actores quieren escuelas más eficaces para solucionar aquellos problemas que interpretan como afectando sus contextos específicos. Los investigadores quieren evitar la interpretación y "los profesores [no sólo los profesores, sino todos los actores] son perfectamente capaces de generar sus teorías personales al estudiar sistemáticamente sus prácticas" (Whitehead y McNiff, 2006: 21). ¿Pero cómo vamos a comparar sistemáticamente dichos contextos diferentes? Esto nos lleva al tercer problema mencionado anteriormente, el problema de dar a las comunidades escolares el poder para definir y evaluar sus factores contextuales y mantener unidades de análisis generalizables.

El problema de dar a las comunidades el poder de definir y evaluar sus factores contextuales y de mantener unidades de análisis generalizables (el MEE desde la perspectiva de los actores). Para continuar con la argumentación de este artículo, hay que señalar que es importante recordar que las desigualdades son reproducidas por dinámicas de poder complejas (GonzálezCasanova, 2004) y que para evitar los estereotipos es necesario permitir a los actores que se definan ellos mismos puesto que son quienes están más familiarizados con las prácticas educativas. Smith (1998) sugiere que los actores deberían establecer las metas y los cambios que se deben perseguir en las escuelas de acuerdo con algunos programas educacionales (y podemos agregar que estos programas también podrían ser evaluados). En la misma línea, Luyten et al. (2005) sugirieron incluir sistemas de retroalimentación de desempeño escolar con el fin de proveer a las escuelas de una retroalimentación sistemática de su desempeño en comparación con otras escuelas. Además, la organización Grupo Académico Children as Decision Makers ${ }^{3}$ (los niños y su participación en la toma de decisiones) actualmente intenta estudiar cómo los niños y estudiantes pueden participar activamente en la toma de decisiones en las escuelas. 
¿Pero cómo vamos a reconocer la agencia de los actores si los programas de investigación los excluyen de los procesos de establecimiento de metas, logros y evaluación? Si el MEE quiere formar parte del debate y de los esfuerzos para luchar contra la reproducción de las desigualdades, parece que tiene que promover la participación y disminuir la exclusión de todos los actores dentro de las escuelas. Al promover la participación de todos, podemos obtener parámetros para ver por lo menos si las escuelas muestran eficacia en la promoción de agencia, que es ampliamente considerado como un elemento de libertad; o para seguir a algunos autores como Michele Scweinsfurth (2007), que han superado algunas dificultades al comparar la educación para el ciudadano global en contextos muy diferentes; o como Rao y Robinson-Pant (2006) que han devuelto totalmente la voz a los actores cuando abordan perspectivas interculturales.

¿Es posible diseñar modelos de eficacia escolar tomando en cuenta a todos lo actores? Pensamos que sí lo es. El gran desafío debe enfrentarse por medio de un enfoque de modelo mixto, ya que los enfoques cualitativo y cuantitativo tienen que complementarse con el propósito de obtener los resultados deseados. Por una parte, la investigación cualitativa puede contribuir a una comprensión más profunda de muchas de las variables relacionadas con la eficacia escolar (especialmente aquellas referidas a los denominados factores contextuales) y de los mecanismos que la causan y que son subyacentes a ella (como las dinámicas de poder). Por la otra, los modelos cuantitativos fácilmente se reproducen a gran escala y son esenciales para establecer unidades de análisis comparables. Como primer paso se debe reconocer, en lo que están de acuerdo los críticos y defensores del MEE, que el MEE necesita fortalecer sus herramientas cualitativas con el fin de contar con mejores unidades de análisis que permitan a las escuelas definir parámetros de eficiencia y que producirían información más adecuada para la construcción de las bases teóricas del programa. La Investigación Acción podría ser una herramienta útil para involucrar a actores en el proceso de investigación. A partir de las categorías de esas investigaciones podríamos establecer algunas unidades de análisis y ver, desde la perspectiva de los actores, cómo las desigualdades y los contextos están siendo afectados por la acción de las escuelas.

La Investigación Acción es una técnica basada en la comunidad que permite que todos los participantes desarrollen su capacidad, "reconoce las limitaciones del conocimiento y la comprensión del investigador 'experto', y toma en cuenta la experiencia y comprensión de aquellos que están involucrados de lleno en el asunto que se explora -los interesados o grupos de interés" (Stringer, 2007: 235). En su vasta producción, Jack Whitehead ha demostrado que los actores pueden investigar su propia práctica, producir sus propias explicaciones 
que pueden ser verificadas constantemente por las respuestas críticas de otros, y lograr validez al "mostrar la autenticidad de la evidencia base, al explicar los estándares de juicio utilizados, y demostrar la racionalidad de lo que afirman (Whitehead y McNiff, 2006: 98).

Sin embargo, algunos autores del MEE podrían sostener que al dar a los actores el poder para definir factores contextuales y evaluar sus cambios, el resultado sería el uso de variables tan diferentes que sería imposible analizarlas o compararlas. Pero la producción de datos cualitativos es una forma común de crear categonías para nuevas investigaciones cuantitativas (Bryman, 2004), y, siguiendo a Habermas (1987), los actores pueden estar conscientes desde el comienzo de la necesidad de lograr algunos acuerdos para conseguir comprensiones inter-subjetivas con el fin de compartir algunas unidades de análisis y discutir aquellos factores que resulte difícil o polémico categorizar en las unidades de análisis acordadas.

Confiar en la agencia y los intereses de los actores, y compartir la responsabilidad en establecer la validez de las variables y su potencial correspondencia con las unidades de análisis, podría dar al MEE nuevos bríos e insertar al programa en los debates actuales acerca de la reducción de las desigualdades y la pobreza por medio de la educación. El enfoque de las capacidades de Sen, el florecimiento humano de Nussbaum, o las perspectivas latinoamericanas tales como la mirada ampliada y crítica de Boltvinik, o las complejas dimensiones de igualdad de Ottone, o muchos otros enfoques, podrían dar algunas señales de las diferentes perspectivas que beneficiarían la eficacia escolar si el MEE participara en un potencial debate acerca de las escuelas eficaces para promover la igualdad.

Además, el trabajo de crear unidades de análisis preliminares no significa empezar de cero. En México, por ejemplo, existen algunos esfuerzos recientes para vincular la eficacia de la educación con aquellas políticas centradas en la reducción de la pobreza (Flores-Crespo y De la Torre, 2007). Como lo han demostrado estudios anteriores, las actuales políticas para luchar contra la pobreza no han producido resultados positivos en los logros académicos aún cuando sean un buen instrumento para aumentar los niveles de matrícula y asistencia escolar (Muñoz-Izquierdo y Villarreal, 2005; Sandoval-Hernández, 2007; Sandoval-Hernández y Muñoz-Izquierdo, 2004).

Ahora, es muy importante reconocer las limitaciones del MEE relacionadas con las desigualdades sociales. A pesar de que sea indiscutible que las escuelas juegan un papel muy importante en disminuir o reforzar las desigualdades, es cierto que en general las escuelas o los sistemas educacionales no pueden 
ser las únicas unidades responsables de la desigualdad social. Tiene que quedar claro que según la definición y las metas del MEE, se puede responsabilizar a las escuelas por el logro de igualdad en la distribución de oportunidades para conseguir resultados escolares iguales entre diferentes grupos de estudiantes. Es altamente probable que al lograr este objetivo se contribuya a la disminución de la desigualdad social; pero este problema está fuera del alcance del MEE.

La igualdad en la educación tiene por lo menos cuatro dimensiones: i) Igualdad de acceso y retención, que se refiere a la noción de que todo niño tenga acceso a la educación y que a ningún niño que desee mantenerse en el sistema educacional se le impida hacerlo, por ejemplo, debido a desventajas económicas. ii) Igualdad de ambiente de aprendizaje, que considera la idea de que todos los individuos gocen de condiciones de aprendizaje equivalentes (por ejemplo, ausencia de sesgo de género en el curriculum). iii) Igualdad de logros escolares, referida a que todos los alumnos dominen habilidades 0 adquieran conocimiento diseñados como metas del sistema educacional en el cual su éxito educacional no dependa de la posición social, económica y cultural de sus familias. iv) Igualdad en el uso de los resultados de la educación, que es la etapa más alta y se refiere a que una vez que los alumnos han dejado el sistema educacional, los individuos o grupos de individuos tengan las mismas oportunidades de usar, en el empleo y en ampliar su vida comunitaria, el conocimiento y habilidades adquiridos.

Como sabemos y como está definido en el programa por los investigadores, el MEE se ubica en la tercera de las dimensiones de igualdad descritas anteriormente. Es indudable que la igualdad es un elemento básico del concepto de eficacia escolar. Una escuela que es eficaz de manera diferenciada (en el sentido de que es 'mejor' para algunos alumnos que para otros) no es una escuela eficaz sino discriminatoria. Es simple, no puede haber eficacia sin igualdad (Murillo, 2003). Sin embargo, lo importante es enfocarse en aquellos aspectos de la igualdad en que el MEE puede contribuir de manera realista, principalmente la igualdad en los resultados escolares.

\section{CONCLUSIONES}

Del análisis de los argumentos presentados en este artículo se puede inferir que:

i) Existen por lo menos tres posturas respecto al debate sobre el MEE. Por razones prácticas las llamaremos la Línea Central, cuyos supuestos básicos se resumen en la sección sobre el núcleo; el enfoque Crítico representado 
por los críticos del MEE cuyos argumentos se encuentran en las secciones referidas a las críticas; y el enfoque Pragmático representado por los investigadores que trabajan en los programas del MEE y cuyos argumentos se presentan en la sección cinturón protector.

ii) Según los criterios para evaluar los programas de investigación contenidos en el marco desarrollado por Lakatos (por ejemplo, la capacidad para mantener la coherencia entre el núcleo y el cinturón protector y para eventualmente conducir a explicaciones innovadoras), el programa MEE no puede ser considerado progresista. Por tanto, se requiere un movimiento en pro de un nuevo programa de investigación con el fin de abordar los principales objetivos establecidos originalmente para el MEE.

iii) Las alternativas actuales son las que ofrecen los enfoques Pragmático y Crítico Realista.

El debate está muy lejos de su término y, como lo admiten Teddlie y Reynols, no se sabe qué valor tendría continuar con el diálogo. Sin embargo, a lo largo del artículo declaramos nuestro acuerdo y desacuerdo con diferentes puntos planteados por cada uno de estos enfoques y también llamamos la atención respecto a tres problemas que deben ser abordados con el fin de lograr producir un nuevo programa de investigación más adecuado para el contexto de América Latina.

En el presente artículo se pone énfasis en la necesidad de evitar la reificación, y se propone adoptar la clarificación de la realidad de Deleuze y Guattari que recurre a la imagen de un rizoma para explicar aquellos aspectos de la realidad que son distinguibles pero están relacionados de manera compleja, entre sí y con las relaciones de poder implicadas. Al utilizar este enfoque, la naturaleza de la escuela está siempre en estado de gestación, y el contexto no se estudia como una realidad externa sino como un artefacto humano que potencialmente puede reproducir y transformar la realidad al transformarse a si mismo.

El principal argumento que se presenta es que el MEE está esencialmente comprometido con la igualdad pero es necesario fortalecer la participación de los actores para evitar la interpretación y reproducción de dinámicas de desigualdad al aplicar el programa. Puesto que los actores son verdaderos expertos, ellos pueden examinar su propia práctica, producir sus propias explicaciones, analizar sus propios argumentos y ser considerados seriamente en la definición de la eficacia escolar. Por tanto, la propuesta aquí presentada es analizar y aplicar el MEE desde la perspectiva de los actores. 
Creemos firmemente que nosotros, como actores en el sistema educacional, no podemos esperar que concluya el debate y luego tratar de importar y adaptar el programa de investigación ganador al contexto latinoamericano. Si queremos avanzar en el mejoramiento de nuestras escuelas y sistemas educacionales, debemos participar en el debate y, al mismo tiempo, generar herramientas teórico-conceptuales desde nuestra propia perspectiva y de acuerdo con nuestros intereses y prioridades, y continuar trabajando con el fin de producir una sólida base para establecer un nuevo programa de investigación que se adecue mejor a la realidad social de América Latina.

\section{NOTAS}

1 Los autores agradecen los comentarios que Hugo Lauder y Kasia Kawalec hicieron a este trabajo ; también damos las gracias al Consejo Nacional de Ciencia y Tecnología CONACYT (México) por los recursos financieros que hicieron posible la realización de esta contribución.

2 El término reconstrucción racional fue acuñado por Lakatos (1970) y se refiere a la reconstrucción de aquello a lo que han estado dedicados lógica e históricamente los investigadores, independientemente de su propio pensamiento, es decir, que no está necesariamente relacionado con los intereses personales de los investigadores ni con sus creencias (Lauder et al., op. cit.).

3 Para mayor información ver http://www.childrenasdecisionmakers.org/ 